\title{
Covalent immobilization of proteins for the biosensor based on imaging ellipsometry
}

\author{
Zhan-Hui Wang, Gang Jin* \\ National Microgravity Laboratory, Institute of Mechanics, Chinese Academy of Sciences, Beijing 100080, PR China
}

Received 11 June 2003; received in revised form 4 December 2003; accepted 5 December 2003

\begin{abstract}
In the development of biosensors, the immobilization of biomolecules at interfaces played a crucial role. The feasibility of using 3-aminopropyltriethoxysilane (APTES) and glutaraldehyde (Glu) to modify silicon surface to immobilize covalently protein for immunoassay with the biosensor based on imaging ellipsometry was investigated. The higher density and stability of human IgG layer could be obtained on the silicon surface modified with APTES and Glu than that on the silicon surface modified with dichlorodimethylsilane (DDS). The human IgG molecules immobilized covalently on APTES-Glu surface bound more anti-IgG molecules than that on DDS surface, which indicated that the human IgG molecules could maintain higher binding capability on APTES-Glu surface. Tween 20 was able to block the undesirable adsorption on APTES-Glu surface, and also enhanced the recognition between human IgG and its antibody on both APTES-Glu and DDS surfaces. The combination of this protein covalent immobilization and the biosensor has the potential to be developed into a fast, simple immunoassay technique.
\end{abstract}

(C) 2004 Elsevier B.V. All rights reserved.

Keywords: Immobilization; Biosensor; Ellipsometry

\section{Introduction}

A concept of biosensor based on imaging ellipsometry was reported several years ago (Jin et al., 1995). The principle of the biosensor is one kind of optical imaging technique that is used to characterize thin film with lateral thickness distribution. A high spatial resolution of about $3 \mu \mathrm{m}$ (laterally) and subnanometer (vertically) can be achieved by the biosensor in

\footnotetext{
* Corresponding author. Tel.: +86-10-6263-1816; fax: +86-106256-1284.

E-mail address: gajin@imech.ac.cn (G. Jin).
}

bioaffinity-based sensing (Jin et al., 1996). It can determine the amount of protein in an adsorbed layer without any labeling like in fluorescence or radioimmunological measurements, which avoids disturbances from conjugated markers or handling with radioactive materials. This allows the application in a broad range of various biological systems. The detection mode with image makes the biosensor possible to detect multiple analytes simultaneously that is highly desirable in biological analysis.

In earlier reports describing the potential of imaging ellipsometry in the field of biomolecular interactions, the biomolecules attached to the surface has in most cases simply been adsorbed to the silicon slides 
prior to measurements (Jin and Wang, 2002; Jin et al., 1996; 1998, 2000; Wang and Jin, 2002).

The use of such attachment techniques involves certain limitations. The immobilized proteins suffer partial denaturation and tend to leach or wash off the surface (Bhatia et al., 1989; Shriver-Lake et al., 1997). In some cases, the adsorbed protein is displaced from the surface by other more active protein, because of the competitive adsorption of proteins (Vroman and Adams, 1969; Vroman et al., 1980). To overcome these problems, covalent immobilization is the preferred method of attaching proteins to silicon surface due to the strong, stable linkage. We investigated the feasibility of using 3-aminopropyltriethoxysilane (APTES) to modify silicon surface and then activating with glutaraldehyde to immobilize protein covalently for the biosensor based on imaging ellipsometry as an alternative to the direct physical adsorption. This protein immobilization method has been used successfully in other immunoassay techniques (Halliwell and Cass, 2001; Lin et al., 1988; Yoshioka and Mukai, 1991; Yuan et al., 2001).

The silicon surface modified with APTES is hydrophobic, because the long aliphatic chains of APTES are hydrophobic moieties and they could promote undesirable adsorption on the surface. To deal with the problem, non-ionic detergents such as Tween 20, Triton X-100 and Nonidet P-40 are generally used as block agents (Zampieri et al., 2000). In this paper, Tween 20 was chosen as block agent, because it cannot only reduce the undesirable adsorption, but also is a potential renaturating agent to improve antigen-antibody binding (Zampieri et al., 2000). The effectiveness of Tween 20 on resisting nonspecific adsorption of proteins on silicon surface modified with APTES was investigated and the renaturating effect of Tween 20 on antigen-antibody interaction was also evaluated by the biosensor in this study.

\section{Materials and methods}

\subsection{Chemicals and materials}

Chemicals used for the buffer (PBS, pH 7.4) preparation were all of analytical grade or better. Aminopropyltriethoxysilane (APTES) and dichlordimethylsilane were purchased from Acros Organics
(Belgium). Glutaraldehyde (50\% aqueous solution), Tween 20 and ethanolamine were purchased from Sigma-Aldrich. Polished silicon slides were purchased from General Research Institute for Nonferrous Metals (China). Water was obtained from a Millipore Milli-Q ion exchange apparatus. Human immunoglobulin $\mathrm{G}$ ( $\mathrm{IgG}$ ) and goat anti-IgG serum were obtained from Sigma (USA). Fetal calf serum was purchased from Hyclone (USA).

\subsection{Cleaning of silicon slides}

The silicon slides were cut into $5 \times 20 \mathrm{~mm}^{2}$ pieces and cleaned with a mixture of $30 \%$ hydrogen peroxide $\left(\mathrm{H}_{2} \mathrm{O}_{2}\right)$ and concentrated sulfuric acid $\left(\mathrm{H}_{2} \mathrm{SO}_{4}\right)$ $(1: 3 \mathrm{v} / \mathrm{v})$ for $30 \mathrm{~min}$. After thoroughly rinsed with water and pure ethanol, the slides were stored in pure ethanol before use.

\subsubsection{Silanization of cleaned silicon slides surfaces with DDS}

The cleaned slides were rinsed three times in trichlorethylene and then placed in a solution of $1 \%$ $\mathrm{v} / \mathrm{v}$ dichlorodimethylsilane (DDS) in trichlorethylene for $5 \mathrm{~min}$ at room temperature. After rinsed three times with trichlorethylene, followed by three rinses in ethanol, the slides were stored in pure ethanol until use. This procedure rendered the slides hydrophobic, with a water advancing contact angle of $80^{\circ}$.

\subsection{Silanization of cleaned silicon slides surfaces with aptes}

The cleaned slides were reacted with a fresh ethanol solution of 3-aminopropyltriethoxysilane (APTES) (5\% APTES, 5\% water and 90\% pure ethanol) for $2 \mathrm{~h}$ at room temperature, followed by rinsing with water three times and pure ethanol three times, and then stored in pure ethanol. This procedure also rendered the surface hydrophobic, with a water advancing contact angle of $50^{\circ}$.

\subsubsection{Physical adsorption of human $\operatorname{Ig} G$ on $D D S$ surface}

The DDS surface was dried in flowing nitrogen and incubated with $0.1 \mathrm{mg} / \mathrm{ml}$ human $\mathrm{IgG}$ solution for various lengths of time at room temperature, and the time necessary to saturate the surface was obtained. 
The surface was then rinsed with water and dried in nitrogen before measured with the biosensor.

\subsection{Covalent immobilization of human $\operatorname{Ig} G$}

The surface silanized with APTES was reacted with a $2.5 \%$ solution of glutaraldehyde in PBS buffer for $2 \mathrm{~h}$, followed by rinsing with PBS buffer. The glutaraldehyde surface was then reacted with $0.1 \mathrm{mg} /$ $\mathrm{ml}$ human $\mathrm{IgG}$ solution at room temperature until the saturated human IgG layer was obtained. The surface was washed with PBS buffer and the remaining aldehyde groups on the surface were deactivated with $1 \mathrm{M}$ ethanolamine for $30 \mathrm{~min}$.

\subsection{Immobilized human $\operatorname{Ig} G$ binding with anti-IgG}

Silicon slides coupled with human $\operatorname{IgG}$ were placed into $0.1 \mathrm{mg} / \mathrm{ml}$ anti-IgG solution with and without the addition of Tween 20 and allowed to incubate for 30 min at room temperature, followed by rinsing with water and dried with nitrogen.

\subsubsection{Blocking nonspecific adsorption with Tween 20}

Silicon slides coupled with human IgG were incubated with fetal calf serum without and with the addition of $0.01 \%, 0.05 \%, 0.1 \%, 0.5 \%, 1 \%, 1.5 \%$ and $2 \%$ Tween 20 for $30 \mathrm{~min}$, respectively. The fetal calf serum was used directly without dilution. After rinsed with water and dried in flowing nitrogen, the surface was measured with the biosensor to determine the amount of nonspecific adsorption.

\subsection{The biosensor setup}

The experiments were carried out with a biosensor based on imaging ellipsometry developed in our laboratory (Jin et al., 2000). The basic experimental setup used in this study was a conventional polarizercompensator-sample-analyzer null exllipsometer. The source was a Xenon lamp, and a specific collimating system was used to provide an expanded parallel probe beam with a diameter of about $25 \mathrm{~mm}$. The beam passed through a polarizer and a compensator (a quarter wave plate) and finally onto the sample at an incident angle of $75^{\circ}$. An optical filter at $633 \mathrm{~nm}$ wavelength was placed in the incident optical passage to select wavelength in order to increase the ellipso- metric contrast of image. The refection beam passed through an analyzer and an imaging lens with a spatial filter located at its focus plane, and then the ellipsometric image was focused on the sensing area of a CCD camera. A digital image was grabbed by and stored in a computer with a grayscale format ( 8 bits, $0-255$ grayscale) for further evaluation by an image processing program. The relationship between the intensity $(I)$ and the thickness $(d)$ of the layer is $I=k d^{2}$, here $k$ is a constant (Jin et al., 1995).

\section{Results and discussion}

\subsection{Surface modification}

There are many methods capable of covalently immobilizing proteins on silicon surface, but it was found that the method used here was very convenient and efficient to perform, since the reaction conditions were very mild with the entire immobilization carried at room temperature. The silicon surface was first activated with APTES, leaving a primary amine group on the surface. Glutaraldehyde (Glu) was subsequently used to react with amine group, yielding an aldehyde that could form an imine linkage with the primary amine group on proteins. In order to compare with physical adsorption, surface modified with DDS was also prepared. The procedures of surface modification rendered the slides hydrophobic, with an advancing contact angle of about $80^{\circ}$ on DDS surface, $50^{\circ}$ on APTES-Glu surface. Catherine M. reported that polymerization of APTES on silicon surface can occur, which leads to heterogeneous surface (Halliwell and Cass, 2001). In this study, the surface modified with APTES was as homogeneous as that modified with DDS measured by the biosensor (images not shown). The polymerization of APTES did not affect the biosensor measurement.

\subsection{Immobilization of human $\operatorname{Ig} G$}

The two surfaces used to immobilize the human IgG were the DDS and APTES-Glu surfaces. The DDS surface was more hydrophobic where there are no functional groups available on the surface to immobilize covalently human $\mathrm{IgG}$, and human $\operatorname{IgG}$ was immobilized on the surface by physical adsorption. 
Hydrophobic interactions were major interactions that resulted in the adsorption of human IgG. The human IgG was covalently immobilized on the APTES-Glu surface by imine linkage formed between the aldehyde group on surface and the primary amine on the human IgG. The human IgG saturated layers on the DDS and the APTES-Glu surfaces were shown in Fig. 1. To be seen easily, the images of the human $\operatorname{IgG}$ saturated layer recorded in grayscale format by computer were translated into three-dimensional images according to the relationship between the intensity and the thickness of the layer. The saturated layer of human IgG on APTES-Glu surface was thicker than that on DDS surface. The absolute thickness of the human IgG saturated layer on APTES-Glu surface was $5.05 \mathrm{~nm}$, and $4.30 \mathrm{~nm}$ on DDS surface measured by ellipsometer.

In order to evaluate the stability of bound human IgG on the two kinds of surfaces, the two surfaces with bound human IgG were placed into PBS buffer and allowed to incubate for $1 \mathrm{~h}$. The results were shown in Fig. 2. About $10 \%$ of the human $\operatorname{IgG}$ desorbed from the DDS surface into PBS buffer. The human IgG immobilized covalently on the APTES-Glu surface was stable in PBS buffer (image not shown). This indicated that the human IgG layer immobilized covalently on APTES-Glu surface was indeed more stable than that immobilized by physical adsorption on DDS surface.

\subsection{Human IgG binding capacity}

The activity of proteins immobilized on solid surface sometimes depends upon the immobilization

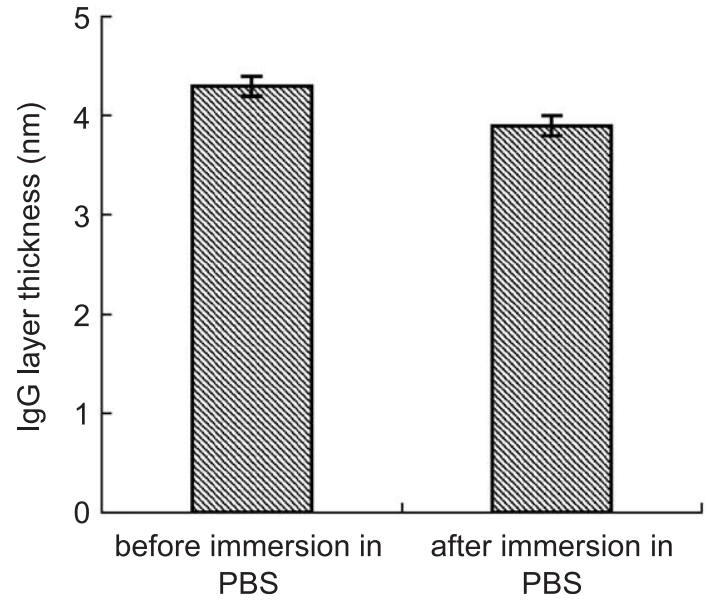

Fig. 2. Stability of immobilized human IgG layer on the DDS surface. About $10 \%$ of the human IgG desorbed from the DDS surface during an immersing in PBS for $1 \mathrm{~h}$.

mode. The interaction between proteins and surfaces may result in protein denaturation. To compare conveniently, the two surfaces coated with human $\operatorname{IgG}$ were incubated into the same concentration anti-IgG solution for the same time. The results were shown in Fig. 3.

Compared with the thickness of the immobilized human IgG layer, the binding of anti-IgG with human IgG caused 1.7 times increase in thickness on APTESGlu surface, and only about 1.0 time on DDS surface. The result indicated that the binding capacity of human IgG immobilized covalently on APTES-Glu surface was larger than that of human IgG immobilized on DDS surface by physical adsorption. One of the main reasons for the reduction of binding activity of human
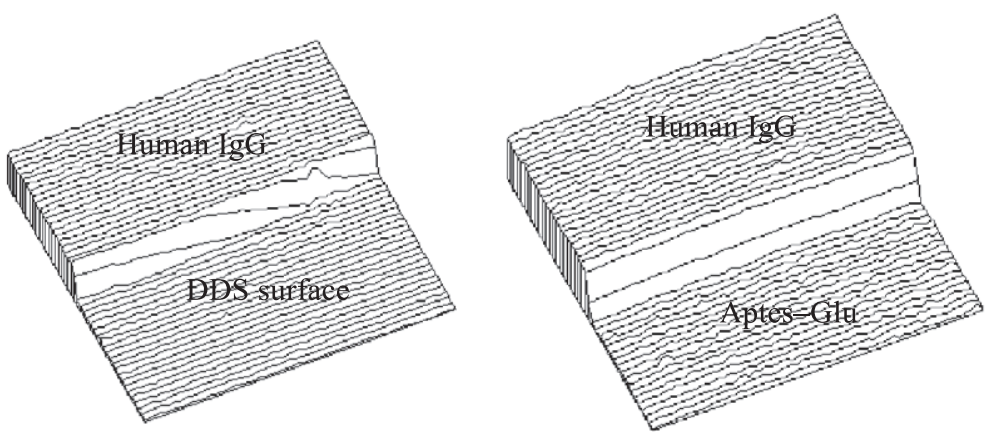

Fig. 1. The saturated monolayers of human IgG on the DDS and the APTES-Glu surfaces. The thickness of human IgG saturated layer on APTES-Glu surface was thicker than that on DDS surface. 


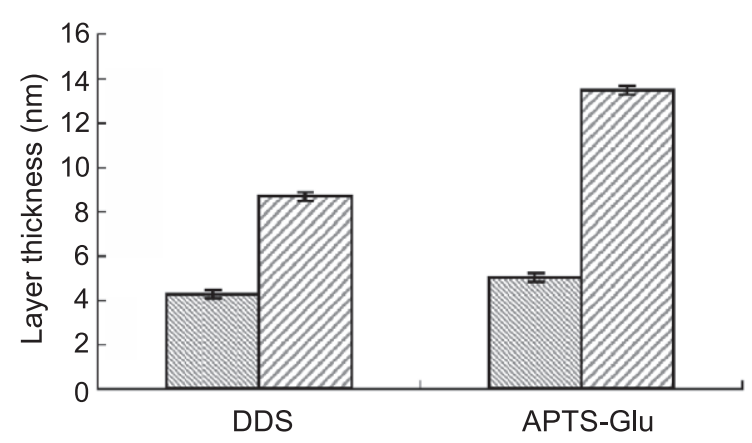

Fig. 3. Layer thickness of human $\operatorname{IgG}$ (圈) and $\operatorname{IgG} /$ anti-IgG complex (ש) on DDS and APTES-Glu surface. The amount of antiIgG bound with human IgG immobilized on APTES-Glu surface was higher than that on DDS surface.

IgG on DDS surface may be due to the interaction between IgG molecules and surface. Protein molecules adsorbed on the surface undergo various transformations caused by the interactions between surface and molecules, which results in the reduction of the biological activity of the protein molecules (Brash and Horbett, 1995). The DDS surface was highly hydrophobic and the hydrophobic interaction between the hydrophobic areas of IgG molecules and surface may result in unfolding of molecules. Some active sites on the $\mathrm{IgG}$ molecule could be denatured during the process. Another possible reason is attributed to steric hindrance of the $\operatorname{IgG}$ molecules on the DDS surface. Some active sites on human IgG molecule were covered because the human IgG molecule was immobilized directly by physical adsorption. The APTES-Glu surface was more hydrophilic than the DDS surface, so the influence of hydrophobic interaction between IgG molecule and surface on the unfolding of IgG molecules was less than that on DDS surface. In addition, the APTES molecule has long aliphatic chain and can serve as a spacer arm for the immobilized human IgG molecule, thus the steric hindrance was also less than that on the DDS surface.

\subsection{Blocking nonspecific adsorption with Tween 20}

The APTES molecule has long aliphatic chain that can promote undesirable adsorption on the surface. In order to overcome the problem, Tween 20 was used as blocking agents. The amount of Tween 20 to block the undesirable adsorption was experimentally determined. The result was shown in Fig. 4. There was indeed some undesirable adsorption on the APTESGlu surface coated with human IgG after incubating with fetal calf serum for $30 \mathrm{~min}$ without Tween 20, but the undesirable adsorption was almost inhibited with the addition of $1 \%$ Tween 20 .

\subsection{Enhancement of anti-IgG binding by Tween 20}

Some authors have reported that Tween 20, in particular, has a renaturalizing effect on antigens, resulting in improved recognition by specific antibodies (Brash and Horbett, 1995). We have introduced Tween 20 into the interaction between human IgG and anti-IgG in order to estimate this phenomenon. The

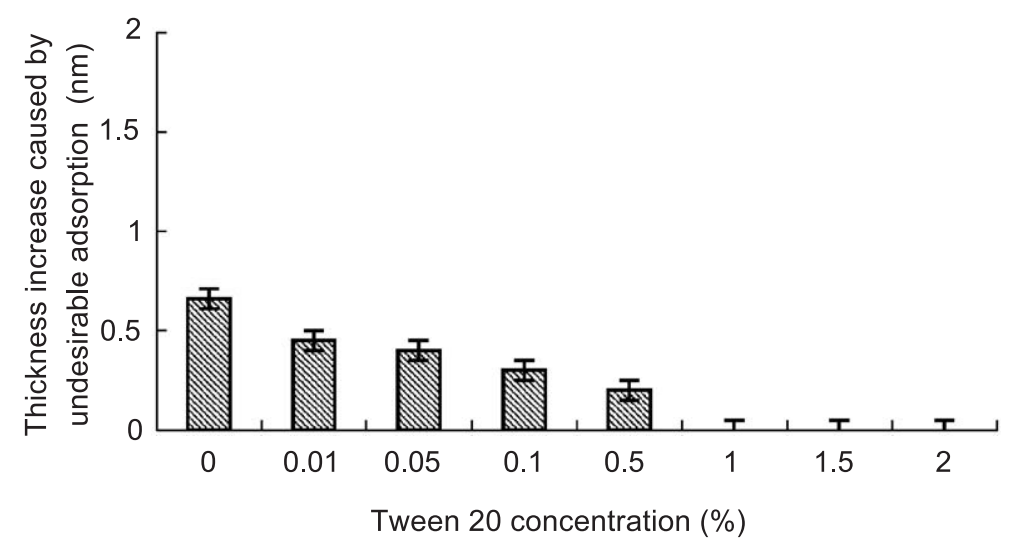

Fig. 4. Blocking with Tween 20 to reduce undesirable adsorption on APTES-Glu surface. The thickness increase was nearly zero when $1 \%$, $1.5 \%$ and $2 \%$ Tween 20 were used. 


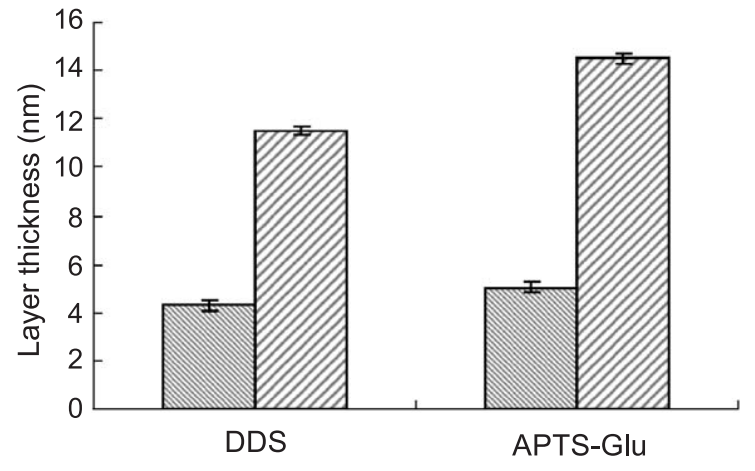

Fig. 5. Layer thickness of IgG (圈) and IgG/anti-IgG complex (四) on DDS and APTES-Glu surfaces. Tween $20(1 \%)$ was added to the buffer during the binding between human IgG and anti-IgG. Tween 20 could improve the recognition between human $\operatorname{IgG}$ and its antibody on both surfaces, the enhancement of anti-IgG binding by Tween 20 was more effective on DDS surface.

APTES-Glu surface and the DDS surface coated with human $\operatorname{IgG}$ were incubated into the anti-IgG solution $(0.1 \mathrm{mg} / \mathrm{ml})$ with the addition of $1 \%$ Tween 20 for 30 min. The results in Fig. 5 showed the binding of the anti-IgG with human $\operatorname{IgG}$ caused about 1.9 times increase in layer thickness on APTES-Glu surface and about 1.6 times on the DDS surface. Compared with the results obtained without Tween 20 , the amount of the anti-IgG bound with human IgG was increased more on DDS surface than that on APTES-Glu surface. The enhancement of anti-IgG binding by Tween 20 might be partly due to the Tween's effect on the surface tension of the dilution buffer, thus decreasing hydrophobic interactions between protein molecule and surface and protein molecule tending to regain its native conformation. The enhancement of anti-IgG binding by Tween 20 was more effective on the human IgG immobilized on the DDS surface by physical adsorption, which suggested that the human IgG molecule conformation was changed larger on highly hydrophobic DDS surface than on the APTES-Glu surface.

\section{Conclusions}

The results above demonstrated the feasibility of using APTES and glutaraldehyde to modify silicon surface to immobilize covalently protein for immunoassay with the biosensor based on imaging ellipsom- etry. The higher density and stability of human IgG layer could be obtained on the silicon surface modified with APTES and glutaraldehyde than that on the silicon surface modified with DDS. The human IgG molecules immobilized covalently on APTES-Glu surface bound more anti-IgG molecules than that on DDS surface, which indicated that the human IgG molecules could maintain their conformation well on APTES-Glu surface. Tween 20 could be used not only as an effective blocking agent to inhibit the undesirable adsorption on APTES-Glu surface, but also as an agent to improve the recognition between antigen and antibody on both APTES-Glu and DDS surfaces.

The biosensor based on imaging ellipsometry is sensitive enough to detect small changes of biomolecular layer in subnanometer. No need to label protein with fluorescence or enzyme, samples can be measured directly. The combination of this protein covalent immobilization and the biosensor has the potential to be developed into a fast, simple and sensitive immunoassay technique.

\section{Acknowledgements}

The national natural science foundation of China and Chinese Academy of Sciences are acknowledged for their supports.

\section{References}

Bhatia, S.K., Shriver-Lake, L.C., Prior, K.J., Georger, J.H., Calvert, J.M., Bredehorst, R., Ligler, F.S., 1989. Use of thiolterminal silanes and heterobifunctional crosslinkers for immobilization of antibodies on silica surfaces. Anal. Biochem. 178, $408-413$.

Brash, J.L., Horbett, T.A., 1995. Proteins at interfaces, proteins at interfaces II. ACS SYMSER 602, 1-23.

Halliwell, C.M., Cass, A.E.G., 2001. A factorial analysis of silanization conditions for the immobilization of oligonucleotides on glass surfaces. Anal. Chem. 73, 2476-2483.

Jin, G., Wang, Z., 2002. Micro-systems for optical protein-chip. Int. J. of Nonlinear Sci. \& Numer. Simul. 3, 191-194.

Jin, G., Tengvall, P., Lundstrom, I., Arwin, H., 1995. A biosensor concept based on imaging ellipsometry for visualization of biomolecular interactions. Anal. Biochem. 232, 69-72.

Jin, G., Jansson, R., Arwin, H., 1996. Imaging ellipsometry revisited: developments for visualization of thin transparent layers on silicon substrates. Rev. Sci. Instrum. 67, 2930-2936.

Jin, G., Zhao, Z.Y., Meng, Y.H., Xing, J.-H., 1998. Visualization of 
molecule interaction between antigen-antibody-one of ellipsometric imaging applications. Proceedings of SPIE-Biomedical Optics and Laser: Diagnositics and Treatment, vol. 3548. SPIE Int Soc Optical Engineering, PO Box 10, Bellingham, WA 98227-0010 USA, Sept. 16-18, 1998, Beijing, China, pp. $131-135$.

Jin, G., Zhao, Z.Y., Wang, Z.H., Meng, Y.H., Xia, L.H., 2000. Optical biosensor with imaging ellipsometry for real-time visualization of bio-molecular interaction. Proceedings of ISIK 2000 Workshop on Biomedical Information Engineering, June 25-27, 2000, Istanbul, Turkey, pp. 269-272.

Lin, J., Herron, J., Andrade, J.D., Brizgys, M., 1988. Characterization of immobilized antibodies on silica surfaces. IEEE Trans. Biomed. Eng. 35, 466-471.

Shriver-Lake, L.C., Donner, B., Edelstein, R., Breslin, K., Bhatia, S.K., Ligler, F.S., 1997. Antibody immobilization using heterobifunctional crosslinkers. Biosens. Bioelectron. 12, $1101-1106$.

Vroman, L., Adams, A.L., 1969. Findings with the recording ellips- ometer suggesting rapid exchange of specific plasma proteins at liquid solid interfaces. Surf. Sci. 16, 438-446.

Vroman, L., Adams, A.L., Fischer, G.C., Munoz, P.C., 1980. Interaction of high molecular weight kininogen, factor XII, and fibrinogen in plasma at interfaces. Blood 55, 156-159.

Wang, Z., Jin, G., 2002. Visualization of the interaction between IL6 and IL-6R by imaging ellipsometry. Chin. J. Biotechnol. 18, 99-101.

Yoshioka, M., Mukai, Y., 1991. Immobilization of ultra-thin layer of monoclonal antibody on glass surface. J. Chromatogr., B, Biomed. Sci. Appl. 566, 361-368.

Yuan, H., Mullett, W.M., Pawliszyn, J., 2001. Biological sample analysis with immunoaffinity solid-phase microextraction. Analyst 126, 1456-1461.

Zampieri, A., Ghirardello, A., Doria, A., Tonello, M., Bendo, R., Rossini, K., Gambari, P.F., 2000. The use of Tween 20 in immunoblotting assays for the detection of autoantibldies in connective tissue diseases. J. Immunol. Methods 239, 1-11. 